Article

\title{
Dynamic Modeling of McKibben Muscle Using Empirical Model and Particle Swarm Optimization Method
}

\author{
Mohd Azuwan Mat Dzahir ${ }^{1,2, *(\mathbb{D})}$ and Shin-ichiroh Yamamoto ${ }^{1}$ \\ 1 Department of Bio-Science Engineering, Shibaura Institute of Technology, 307 Fukasaku, Minuma-ku, \\ Saitama-City, Saitama 337-8570, Japan; i045289@shibaura-it.ac.jp \\ 2 School of Mechanical Engineering, Faculty of Engineering, Universiti Teknologi Malaysia, Skudai 81310, \\ Johor Bahru, Malaysia \\ * Correspondence: azuwan@utm.my; Tel.: +60-107153384
}

Received: 9 May 2019; Accepted: 19 June 2019; Published: 21 June 2019

check for updates

Featured Application: Robotic equipment, humanoid robots, rehabilitation orthotics, industrial automation and biological system.

\begin{abstract}
This paper explores empirical modeling of McKibben muscle in characterizing its hysteresis behavior and nonlinearities during quasi-static, quasi-rate, and historic dependencies. The unconventional materials-based actuating system called McKibben muscle has excellent properties of power-to-weight ratio, which could be used in rehabilitation orthosis application for condition monitoring, physical enhancement, and rehabilitation therapy. McKibben muscle is known to exhibit hysteresis behavior and it is rate-dependent (the level of hysteresis depends closely on rate of input excitation frequency). This behavior is undesirable and it must be considered in realizing high precision control application. In this paper, the nonlinearities of McKibben muscle is characterized using empirical modeling with multiple correction functions such as shape irregularity and slenderness. A particle swarm optimization (PSO) method is used to determine the best parametric values of the proposed empirical with modified dynamic friction model. The LabVIEW and MATLAB platforms are used for data analysis, modeling and simulation. The results confirm that this model able to significantly characterize the nonlinearities of McKibben muscle while considering all dependencies.
\end{abstract}

Keywords: McKibben muscle; empirical modeling; particle swarm optimization

\section{Introduction}

McKibben muscle plays an important role in the development of assistive rehabilitation robotics system. The development of rehabilitation orthosis, which implemented McKibben muscle, is rather slow when compared to other types of actuated rehabilitation orthosis using ac-motor, dc-motor, pneumatic cylinder, linear actuator, series elastic actuator (SEA), and brushless servomotor. However, due to its clear advantages such as low weight, structural flexibility, compactness, and inherent compliance compared to other types of artificial muscles, the researcher's interest in this field has grown exponentially in past decade [1-3]. This growth also due to the challenges it provides in resolving nonlinearity behaviors and difficulties in controlling such systems. The inherent nonlinearity behaviors of McKibben muscle present because of nonlinear relationship in between its contracting force, contracting length and pressure [4-6]. The other factors include nonlinearity behaviors of pressure build-up and hysteresis due to its geometric construction and frictions. Various approaches and methods have been introduced to reduce the effect of hysteresis, where a precise control system is desired. In addition, numerous control strategies were considered to resolve nonlinearity problems in 
both static and dynamic modeling. Throughout the years, a number of physic and phenomenological approaches had been considered in static modeling such as force generation equation, empirical model, conservation of energy (virtual work), polynomial-based model, and Hill's muscle model [1-39]. However, empirical modeling was often used to characterize the behaviors of McKibben muscle compared to others due to previous achievement [7-27]. This is also similar for the virtual work theorem which can be related with empirical model [28]. On contrary, dynamic modeling of McKibben muscle also have been evolved rapidly with implementation of various models such as coulomb friction and viscous friction $[17,18]$.

In static modeling of McKibben muscle using empirical model, it was first introduced by K. Inoue in 1988 [7]. After that, B. Tondu, in 1994, imposed similar approach and modified the model with empirical constant $k$ to account for end deformation of the muscle [8]. These early researches became the pillar of other researches and major contribution in this research field as DB. Reynolds in 2003 introduced empirical dynamic force model with parallel arrangement of a spring element, damping element, and contractile force element [9]. While, A. Hildebrandt, in 2005, improved the empirical model with force modeling and considered a one-way cylinder that moves against a spring [10]. The pulling forces of the muscles were approximated by polynomial functions. Meanwhile, S. Davis, in 2006, improved the empirical model and included braid effects on contractile range [11,12]. Then, analyses its frictional effect to produce a more accurate model and removed the necessity of correction parameter $k$. Contrarily, T. Kerscher, in 2006, proposed a combination of empirical-geometric model with a variable spring and parallel variable damper system [13]. Due to its nonlinearities, the static characterization of McKibben muscle becomes more complicated with introduction of these compensation methods. To reduce the complexity, KC. Wickramatunge, in 2009-2010, introduced empirical modeling using spring system with variable stiffness of parameter $\mathrm{K}$, where the parameter was taken as 2nd Order of Polynomials (OP) of pressure and length [14,15]. Then, S.V. Krichel, in 2010, improved the force modeling of McKibben muscle and considered one-way cylinder which moves against a spring. The pulling forces of the muscles were approximated by polynomial functions [16]. As suggested by B. Tondu in 2000, BS. Kang in 2009 and T. Itto in 2011 also considered implementation of correction factor $q>1$ for shape irregularity $[17,18]$. The correction factor $q$ is a pressure dependent parameter of exponential function. Afterwards, J. Sarosi, in 2012-2015, improved the existing empirical model proposed by B. Tondu and T. Kerscher with 5-6 parameters models $[19,20]$. On contrary, M. Tothova in 2013 proposed an advanced geometric muscle model where changes of the pressure and diameter of the muscle can be varied.In addition, volume of cylinder was expressed as function of muscle diameter and valve diameter [21]. Similar to this work, Z. Kulesza, in 2014, also considered the same issues where the effects of the end-cap diameters were based on cone shapes [22]. On the other hand, T. Nuchkrua in 2014 evaluated the static muscle force based on empirical stiffness to actuate the muscle actuator via hydrogen absorption/desorption [23]. Inspired by the R.B. Reynold model, B. Yao in 2016 proposed a synthetically empirical model consisted of hysteresis, viscous damping, rubber elasticity and contractile elements in parallel [24]. Recently, A.A. Ibadi, in 2016-2018, also considered an exponential correction factor $q$ to account for shape irregularities of McKibben muscle [25-27]. These researches were focused on quasi-static empirical modeling without quasi-rate and historic dependencies. Eventhough, S. Davis considered both quasi-static and historic dependencies by creating minor hysteresis loops. Furthermore, B. Yao contemplated quasi-static, quasi-rate, and historic dependencies in their model. In addition, T. Kerscher used correction factor $\mu(\varepsilon)$ to compensate the asymmetric behavior of McKibben muscle at low and high pressure; while K.C. Wickramatunge implemented the 2nd OP equations within the region. Similarly, J. Sarosi introduced empirical model with 5-6 parameters to handle this asymmetric region during low and high input pressure. However, most of others researchers just implemented intelligent controllers such as PID, Cascaded, Tracking, Fuzzy Logic, and Fuzzy Adaptive PID to compensate the nonlinearities of McKibben muscle.

On the other hand, dynamic modeling of hysteresis considers the output's delay which happened during cyclic contraction and expansion of McKibben muscle. This is due to frictions in between 
thread-to-thread and thread-to-bladder of the McKibben muscle including other unidentified factors. When compared static and dynamic models of McKibben muscle, asymmetric hysteresis offset can be observed. To resolve this behavior, K. Inoue, in 1988, proposed a model consisted of frictional term proportional to the angular velocity of the pulley and spring term proportional to the pulley angle [7]. Then, CP. Chou, in 1996, implemented static coulomb friction model and create static offset during both contraction and expansion stages. While, B. Tondu, in 2000, proposed and compared the implementation of static and dynamic coulomb friction models [28,29]. Afterwards, R.W. Colbrunn, in 2001, resolved the problem by using three elements models of spring, viscous damper and coulomb friction [30]. On contrary, J. Schroder, in 2003, implemented static offset of force which is practically similar to the coulomb friction model [31]. On the other hand, S. Balasubramanian in 2006 considered the dynamic modeling through the FLP graph implementation [32]. Following B. Tondu, S. Davis, in 2006, introduced dynamic coulomb friction model. However the proposed empirical model neglected the $k$ factor [12]. Furthermore, B.S. Kang, in 2009, implemented both velocity's dependence coulomb and viscous friction models [17]. While, V. Jouppila in 2010 applied static coulomb friction model as proposed by CP. Chou and B. Tondu [33]. Then, T. Itto in 2011 introduced a spring element under no pressure condition into coulomb and viscous friction models proposed by BS. Kang based on vertical load system [18]. In addition, R. Tang in 2012 improved the hysteresis modeling using spring, viscous damper, and dynamic coulomb friction model as proposed by R.W. Colbrunn [34]. Meanwhile, J. Zhong, in 2014, investigated the effect of strand on strand and bladder on bladder friction models for the dynamic modeling [35].

The above mentioned approaches without a doubt were the pillar in hysteresis modeling of McKibben muscle on both static and dynamic modeling. Through some review analysis and simulation of the existing empirical models, significant research gap has been found to improve dynamic modeling of McKibben muscle. As suggested by B.S. Kang and T. Itto on dynamic modeling of hysteresis, the hysteresis behavior is characterized using both velocity's dependence coulomb and viscous friction models. However, in vertical load system it is found that friction on the McKibben muscle depends on both input excitation frequency and vertical load. Thus, additional correction functions $\gamma(F)$ and $\gamma(f)$ for compensating hysteresis behavior were introduced to improve adaptability of the proposed empirical model while considering quasi-static, quasi-rate, and historic dependencies. The critical parameters of the proposed empirical model were optimized using particle swarm optimization (PSO) method.

\section{Materials and Methods}

Two McKibben muscles with initial lengths of $300 \mathrm{~mm}$ and $530 \mathrm{~mm}$ were prepared for data collection. The data was used for characterizing nonlinearities and hysteresis phenomena of McKibben muscle. The McKibben muscle consists of a rubber tube, braided mesh sleeve, and connectors. Experimental tests were performed using a vertical load system as shown in Figure 1. It consists of McKibben muscle, displacement sensor, load sensor, accelerometer, static load, regulator, compressor, processor, and data acquisition. A graphical user interface (GUI) for operating vertical load system was prepared using LabVIEW software (Academic Software-Spring 2015, National Instruments Corporation, Ireland). The input pressure of McKibben muscle was generated using random wave signal to create cyclic motion of contraction and expansion. This random wave was used for both quasi-static and quasi-rate dependency tests. In addition, this random wave also historic dependence where it creates minor hysteresis loops with nonlocal memory behaviors. The approach implemented in this research paper emphasized empirical modeling based on law of physic and geometrical analysis of McKibben muscle. To compensate the quasi-static behavior of McKibben muscle, shape irregularities $q(P)$, and slenderness $\mu(\varepsilon)$ correction functions were used for static modeling. To develop a model while considering multiple dependencies, it will require for another empirical modification. Therefore, additional correction functions were proposed to compensate dynamic irregularities of McKibben muscle. Where, the modification was made to generate dynamic friction model. The analysis and 
characterization of the hysteresis data were programmed with MATLAB language (MATLAB R2017a, MathWorks Inc., academic use); while the MATLAB Simulink was used to simulate existing and proposed empirical models. In addition, Particle swarm optimization (PSO) method was implemented to obtain best parametric values of the empirical model. Finally, model testing and correlation test were performed to analyze the performance of the proposed model.

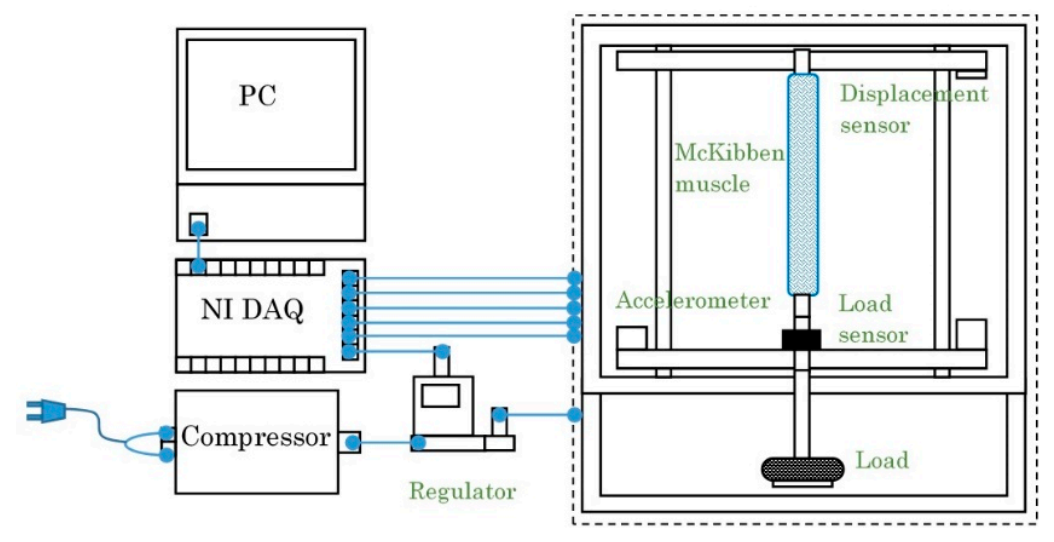

Figure 1. Vertical load system.

\section{Hysteresis Phenomena}

The research on hysteresis phenomenon has been recorded for decades. Early study on this phenomenon is first observed in 1881 by James A. Ewing within the field of ferromagnetism [36]. It is historic dependence, where it can be considered as a system that has memory function. The effects produce from the input to the system are reflected with a certain delay in time. According to research conducted by $\mathrm{Oh}$ and Bernstein in 2005, hysteresis is a quasi-static phenomenon in which a sequence of periodic inputs produces a nontrivial input-output loop as the period of input increases without bound [37]. This phenomenon arises in diverse fields ranging from physics to biology, from material science to mechanics, and from electronics to economics [38,39]. The focus in this paper is on the inherent hysteresis phenomena in McKibben muscle. The hysteresis phenomena in McKibben muscle have more asymmetry and can be difficult to model when compared to other fields such as smart actuators, material, ferromagnetism, etc. This is especially at low and high input pressure region where the contraction length behavior is highly nonlinear.

\section{Hysteresis Data Characterization}

The structural materials of McKibben muscle inherently lead to hysteresis phenomena during cyclic contraction and extension. This nonlinearity is addressed as a difficult error source that needs to be handled, especially in a precise control system. The major and minor hysteresis loops can be visibly seen by conducting either an isometric or isotonic tests using vertical load system. As describe in Section 2, the experimental tests were conducted for both quasi-static and quasi-rate dependencies. For quasi-static test, McKibben muscle was vertically attached with different static loads $(0-300 \mathrm{~N})$. The change in static load produces different set of hysteresis data. Where, the maximum contracting length reduced with increased of static load. For quasi-rate test, the McKibben muscle was induced with random wave signal (input pressure) and its frequency was varied in between 0.5 and $2.0 \mathrm{~Hz}$. This test was performed because level of hysteresis depends closely on the rate of input excitation. In addition, the change in velocity also causes the friction between the rubber tube and braided mesh sleeve to significantly change, and thus produce a different set of hysteresis data. The desired output from the vertical load system was the contracting length of McKibben muscle. Therefore, a laser displacement sensor was used to measure the contracting length of McKibben muscle during cyclic contraction-expansion. Figure 2 shows two set of hysteresis data obtained from vertical load test. The hysteresis data reflects quasi-static, quasi-rate, and historic behaviors of the McKibben muscle. 
In addition, both major and minor hysteresis loops can be visibly seen from the Figure 2 . The major hysteresis loop can be seen as an outer layer of the hysteresis data; while the minor hysteresis loop shows the nonlocal memory behavior of McKibben muscle. This behavior has historic dependence, where the hysteresis behavior depends on the previous values of force, length, and pressure. The two sets of McKibben muscle also produce similar hysteresis data, which clarified the consistency of identical McKibben muscle's nonlinear behaviors.
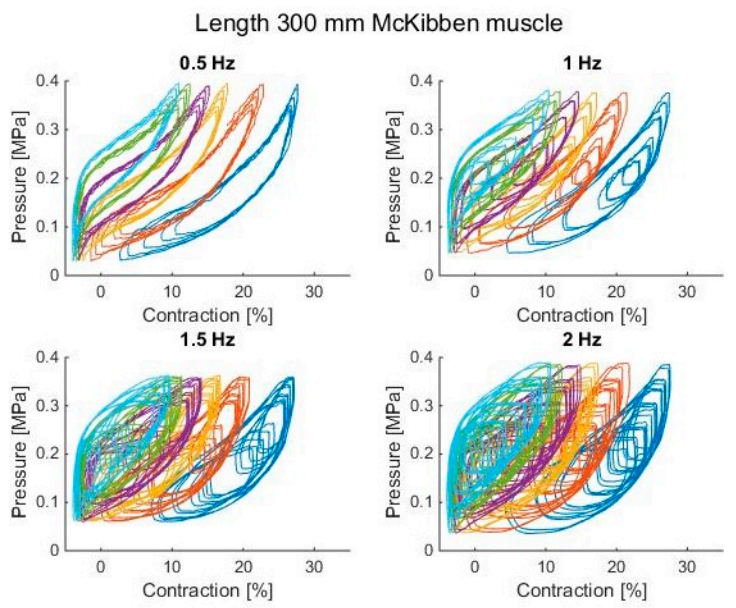

(a)
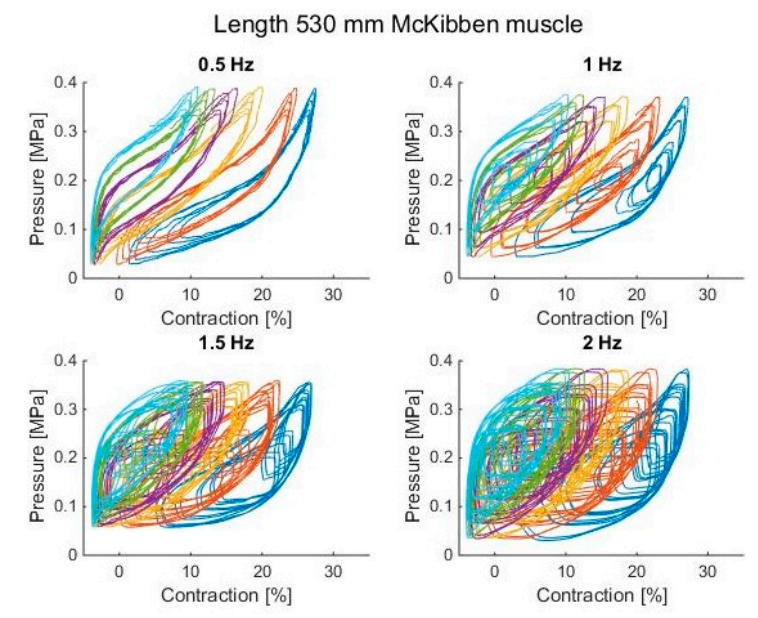

(b)

Figure 2. Hysteresis data at all dependencies: (a) McKibben muscle of $300 \mathrm{~mm}$ and (b) McKibben muscle of $530 \mathrm{~mm}$.

\section{Parametric Identification of the McKibben Muscle Model}

The parametric estimation can be considered in two different points of views. Firstly, an identifier can be designed and substituted into the model of the system. It will imitate the behavior of the real system as nearly as possible with a minimum error produce. This identification technique is known as nonparametric identification. Secondly, parameters of the proposed model can be estimated through an optimization tool. This identification technique is known as parametric identification. In this technique, the system parameters can be estimated using several methods such as least mean square, recursive least square, genetic algorithm and particle swarm optimization [38,39]. Recently, particle swarm optimization method has become major contributions within the control application because of its powerful application. The purpose of the system identification is to locate best parametric solution of dynamic system based on the provided input and output data. Previous studies have applied various techniques to solve the problem related to system identification [40-42].

\section{Particle Swarm Optimization}

Recently, research area that has been receiving an increase attention by the research community, as well as the industry is the optimization algorithm [40]. It is a procedure or numerical method used to find the maximum or the minimum of a function operating with certain constraints [41]. One of the most reliable optimization methods is particle swarm optimization (PSO). It is a computational algorithm technique based on swarm intelligence. This method is inspired based on the observation of social interaction and animal behaviors (i.e., fish schooling and bird flocking). It imitates the way they search for food by emphasizing cooperation and the competition among the entire group population [42]. A swarm consists of individuals, which is called particles and it is initialized with a population of random solutions; where each of these particles represents a different possible set of the unknown parameters to be optimized. The particles will fly around in a multidimensional search space, and then adjust their position according to its own experience and the experience of its neighboring particle [43]. It is designed to efficiently search the solution space by swarming the particles towards 
the best fitting solution encountered in previous iterations. This, eventually, will be approaching better solutions through the course of the process; then finally converging on a single minimum or maximum solution [44]. The performance of each particle is determined based on a predefined fitness function of the problem. Actually, the implementation of PSO has been reported in many research works in this field. Besides, PSO has been regarded as a promising optimization algorithm due to its simplicity, low computational cost, and good performance [45,46].

To describe the PSO algorithm, it is initialized with a population of random solutions. These random solutions are called particles, and each of the potential solutions is also assigned with a randomized velocity function [47]. The PSO relies on the information exchange in between particles of the population called swarm. The particles will adjust their trajectories towards their current best solution (fitness), $P_{\text {best }}$. In addition, each particle modifies its trajectory towards the previous best position, $G_{b e s t}$, attained by any member of its neighborhood as shown in Figure 3. Furthermore, each particle also moves in the search space with an adaptive velocity function. The measurement of fitness evaluates the performance of each particle to determine if the best fitting solution is achieved. During the process, the fitness of the best individual will improve over time and eventually tends to stagnate towards the end of the process; where the stagnation of the process coincides with the successful discovery of the global optimum.

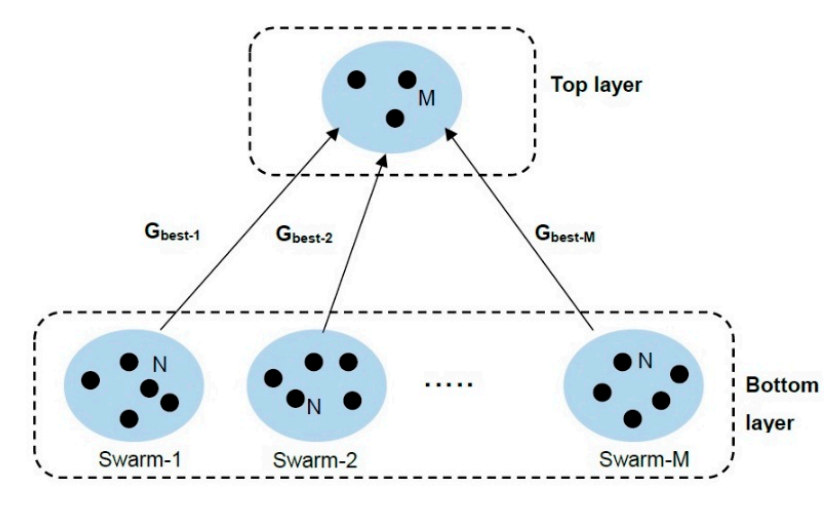

Figure 3. Particle swarm optimization (PSO) block diagram.

Several parameters such as velocity constant, population size, and number of iterations need to be defined before the process. These parameters will decide to a great extent the ability of global minimization. Where, the maximum velocity affects the ability of escaping from local optimization and refining global optimization. Then, the size of swarm stabilizes the requirement of computational cost and global optimization. Table 1 shows the initialized values for the selected parameters. The optimization parameter is realized either when the maximum number of iterations increased or with an attainment of satisfactory fitness value. The fitness value is a reciprocal of the magnitude of the objective function where minimization of objective function was considered. In this paper, the termination criteria considered with the attainment of satisfactory fitness value, which occurred with the maximum number of 10 generations. For each generation, the best among the 50 particles is considered as a potential solution.

Table 1. Initialized value for selected parameters.

\begin{tabular}{ll}
\hline Population size, $N$ & 50 \\
Number of generations, $G$ & 10 \\
Velocity constant, $v_{1}$ & 1.5 \\
Velocity constant, $v_{2}$ & 1.5 \\
\hline
\end{tabular}




\section{Empirical Modeling of the McKibben Muscle}

Generally, there is no fundamental theory that allows for a general mathematical framework for modeling the hysteresis effects because the origins of these phenomena are often unclear and complicated [48,49]. It can be observed from literature that most of existing hysteresis models are initially developed to describe a particular type of hysteretic system. However, its mathematical form could be implemented for multidisciplinary extension. Most of the approaches used in characterizing the hysteresis behavior are either by the law of physics or the phenomenological method [50]. For example, empirical model is established based on the principle of physics, such as the relationships of energy, displacement, and geometric configuration [39]. However, it is not easy to describe a model by this principle because the physical feature of a hysteretic system is usually very complicated. In addition, the empirical model developed for one McKibben muscle may not be used for another kind of natural compliant or soft actuator, and thus no model generalization is possible. With the availability of various physic based models, a complete model analysis was carried out to determine unsettled problem which remains in the hysteresis characterization on both static and dynamic modeling of McKibben muscle using empirical model.

\subsection{Contracting Force Model}

The static modeling of hysteresis was used to configure the contracting force of the McKibben muscle with various pressures and contracting lengths. Several modifications on empirical modeling have been suggested after it was first introduced by K. Inoue in 1988 [7]. The first modification model included an empirical constant $k$ to account for end deformation of the muscle as suggested by $\mathrm{B}$. Tondu in 1994, as shown in Equations (1) and (2) [8].

$$
\begin{gathered}
F_{\mathcal{S}}(P, \varepsilon)=\frac{\pi D_{o}^{2} P}{4}\left(\frac{3(1-k \varepsilon)^{2}}{\tan ^{2} \alpha_{o}}-\frac{1}{\sin ^{2} \alpha_{o}}\right) \\
k=c_{1}
\end{gathered}
$$

The second modification model included a shape irregularity correction function $q$ which tunes the slope of the considered static contracting force model as suggested by B. Tondu in 2000, B.S. Kang in 2009, and T. Itto in 2011 [17,18,25]. The shape irregularity was defined as an exponential function of input pressure as shown in Equations (3) and (4).

$$
\begin{gathered}
F_{s}(P, \varepsilon)=\frac{\pi D_{o}^{2} P}{4}\left(\frac{3(1-q(P) \varepsilon)^{2}}{\tan ^{2} \alpha_{o}}-\frac{1}{\sin ^{2} \alpha_{o}}\right) \\
q(P)=c_{1}+c_{2} e^{c_{3} P}
\end{gathered}
$$

The third modification model included both correction functions of shape irregularity $q$ and slenderness $\mu$ as suggested by T. Kerscher in 2006 [13]. The slenderness was defined as an exponential function of contracting length as shown in Equations (5)-(7). Its implementation also considers the various length of McKibben muscle; where the contracting force value will significantly increase with increment in McKibben muscle's initial length.

$$
\begin{gathered}
F_{\mathcal{S}}(P, \varepsilon)=\mu(\varepsilon) \frac{\pi D_{o}^{2} P}{4}\left(\frac{3(1-q(P) \varepsilon)^{2}}{\tan ^{2} \alpha_{o}}-\frac{1}{\sin ^{2} \alpha_{o}}\right) \\
q(P)=1+c_{1} e^{c_{2} P} \\
\mu(\varepsilon)=c_{3} e^{c_{4} \varepsilon}+c_{5}
\end{gathered}
$$




\subsection{Extracted Hysteresis Model}

Two rules can be distinguished in dry friction between two contacting surfaces which are presliding and sliding. The extracted hysteresis can be noticed to exhibit similar behavior when compared with presliding or sliding regimes of mechanical friction. In the presliding rule, the adhesive forces are more dominant at asperity contacts with rate independency; where the friction force becomes dependent to function of displacement rather than the velocity. Meanwhile, the sliding regime happens when the displacement increases until the asperity contacts are broken away [51]. In this paper, both coulomb and viscous frictions are considered as the hysteresis phenomena source. The dry coulomb friction term involves the hysteresis behavior due to the thread-on-thread and thread-on-bladder friction of the McKibben muscle. This coulomb friction is a static friction which switches during cyclic contraction and expansion process [17]. Its mathematical form is shown in Equation (8). The mathematical form helps to predict the direction and magnitude of the friction force in between two bodies with dry surfaces in contact.

$$
F_{c c}(\dot{\varepsilon})=c_{c} \operatorname{sign}(\dot{\varepsilon})
$$

The viscous friction term describes a viscosity of the compressed air and the friction between the air and the tube's inner surface as shown in equation (9). There are two parts of viscous friction. The first part contributes from the viscosity of the compressed air itself. In other words, it is the frictional effects between gas particles. While the second part of viscous friction contributes from the friction between the compressed air and the inner wall of the McKibben muscle's rubber tube [17,18].

$$
F_{v c}(\dot{\varepsilon})=c_{v} \dot{\varepsilon}
$$

Modification was required to improve the adaptability of the model while considering quasi-static, quasi-rate, and historic dependencies. This modification requests additional correction parameters for load-dependent $\gamma(F)$ and rate-dependent $\gamma(f)$ in developing empirical modeling with dynamic friction model. The mathematical formulation of the dynamic friction model is shown in Equations (10) and (11). Both correction functions were designed as a first-order equation of static load $(\gamma(F)=\mathrm{a} F)$ and excitation frequency $(\gamma(f)=b f)$.

$$
\begin{gathered}
c_{v}=\gamma_{F} \gamma_{f} \\
F_{h y s}(\dot{\varepsilon})=c_{c} \operatorname{sign}(\dot{\varepsilon})+\gamma_{F} \gamma_{f} \dot{\varepsilon}, \varepsilon \neq 0
\end{gathered}
$$

\subsection{Elasticity Model}

The McKibben muscle behaves asymmetrically at low pressure especially when the muscle was further stretched, even after reaching its maximum expansion; where the mechanism of McKibben muscle only consisted of braided thread and a rubber tube [18]. Thus, in this state, the McKibben muscle is considered to be an elastic body as the expansion reached zero internal pressure, as shown in Equation (12). This condition was implemented to modify the dynamic force of McKibben muscle; where there is no contraction force generated which caused no force to balance the vertical load.

$$
F_{\text {elastic }}=K\left(L_{o}-L(1-\varepsilon)^{3}\right), \quad \varepsilon \leq \frac{L-L_{o}}{L}
$$

\subsection{Dynamic Equation for Vertical Load System}

Most of the applications based on McKibben muscle involve direct load to the system, which is required for lifting and sustaining a solid body. Therefore, in this paper, the dynamic behavior of the vertical load system was considered within the mathematical formulation. When the static load is pulled up due to contracting force generated from the McKibben muscle, it causes friction between the thread and bladder of the McKibben muscle. As suggested in previous section, the dynamic friction model was affected with changes in static load and input excitation's frequency. The equation 
generated for the force dynamic of the vertical load system was properly described and shown in Equations (13)-(15). The implementation of these equations were illustrated as block diagram shown in Figure 4. Meanwhile, the description of model parameters and parameters optimized by PSO algorithm were shown in Table 2.

$$
\begin{gathered}
F_{d y n}=F_{s}(P, \varepsilon)-M g-F_{h y s}(F, \dot{\varepsilon}) \\
F_{d y n}=M L \ddot{\varepsilon} \\
F_{d y n}=F_{n e t}(P, \varepsilon)-F_{h y s}(F, \dot{\varepsilon}), \quad \varepsilon>\frac{L-L_{0}}{L} \\
F_{d y n}=F_{n e t}(P, \varepsilon)-F_{\text {elastic }}-F_{\text {hys }}(F, \dot{\varepsilon}), \quad \varepsilon \leq \frac{L-L_{0}}{L}
\end{gathered}
$$

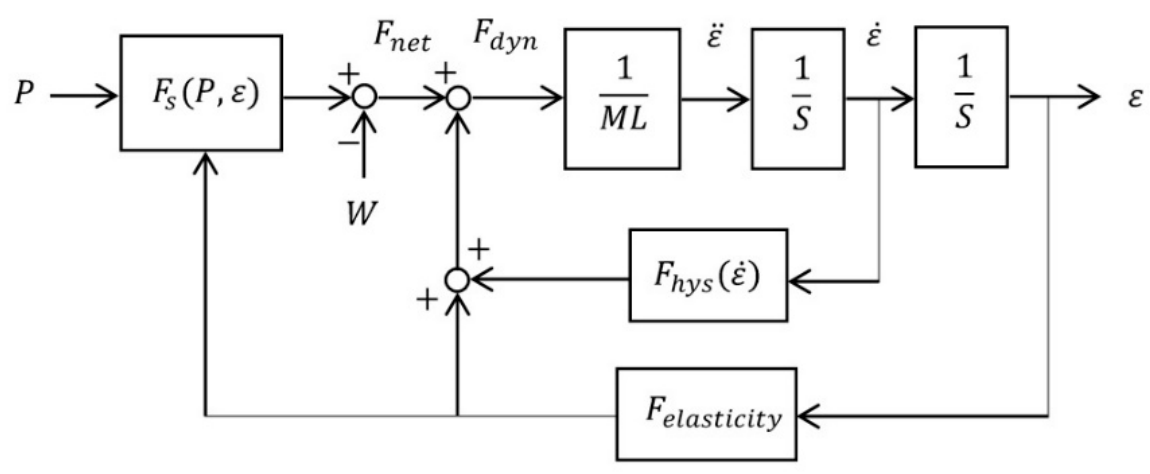

Figure 4. Block diagram of empirical modeling.

Table 2. Model parameters.

\begin{tabular}{clcl}
\hline Variable & \multicolumn{1}{c}{ Description } & Variable & \multicolumn{1}{c}{ Description } \\
\hline$P$ & Input pressure & $\gamma_{f}$ & $\begin{array}{l}\text { Rate dependency } \\
\text { correction function }\end{array}$ \\
$\varepsilon, \dot{\varepsilon}, \ddot{\varepsilon}$ & $\begin{array}{l}\text { Contracting length, velocity and } \\
\text { acceleration }\end{array}$ & $F_{d y n}$ & Dynamic force \\
$\alpha_{0}$ & Initial braid angle & $F_{s}$ & Static force \\
$M$ & Mass of the weight & $F_{h y s}$ & Hysteretic force \\
$g$ & Gravitational acceleration & $F_{c c}$ & Coulomb friction \\
$D_{o}$ & Initial diameter & $F_{c v}$ & Viscous friction \\
$L_{0}, L$ & Initial length and length & $F_{n e t}$ & Net force \\
$K$ & Coefficient of elasticity (PSO) & $F_{\text {elastic }}$ & Elastic force \\
$c_{1}, c_{2}, c_{3}, c_{4}, c_{5}, c_{6}$ & Constant coefficients (PSO) & $F$ & Number of population \\
$c_{c}, c_{v}$ & Coulomb and viscous friction coefficients & $G$ & Number of generation \\
$q$ & Shape irregularity correction function & $v_{1}, v_{2}$ & Velocity constants \\
$\mu$ & Slenderness correction function & $P_{b e s t}$ & Local optimum \\
$\gamma_{F}$ & Load dependency correction function & $G_{b e s t}$ & Global optimum \\
\hline
\end{tabular}

\section{Results}

The results were divided into subsections to properly describe the flow of the modeling progress from data collection until model verification. The subsections include force dynamic characteristics, empirical model simulation, and empirical model testing, while considering quasi-static, quasi-rate, and historic dependencies. While the results consisted of experimental hysteresis data, empirical modeling, model simulation, parametric optimization, and model testing.

\subsection{Force Dynamic Characteristics}

Figure 5 shows the force dynamic characteristics of the proposed empirical modeling at various static loads and input pressures. Experimental contacting force data $\left(F_{\text {exp }}\right)$ was obtained through 
actual testing of McKibben muscles using the vertical load system. While a static contracting force $\left(F_{S}\right)$ illustrates the graphical solution from the empirical modeling. This static force remains unchanged, which indicates the actual force characteristic of the McKibben muscle with zero load implementation. Net contracting force $\left(F_{n e t}\right)$ and dynamic contracting force $\left(F_{d y n}\right)$ show the dynamic behavior of the vertical load system. Where the static contracting force was affected by the static load and frictional effects of the contacting surface in between rubber tube and braided mesh sleeve, thus yielding to the hysteresis phenomena during cyclic contraction and expansion.

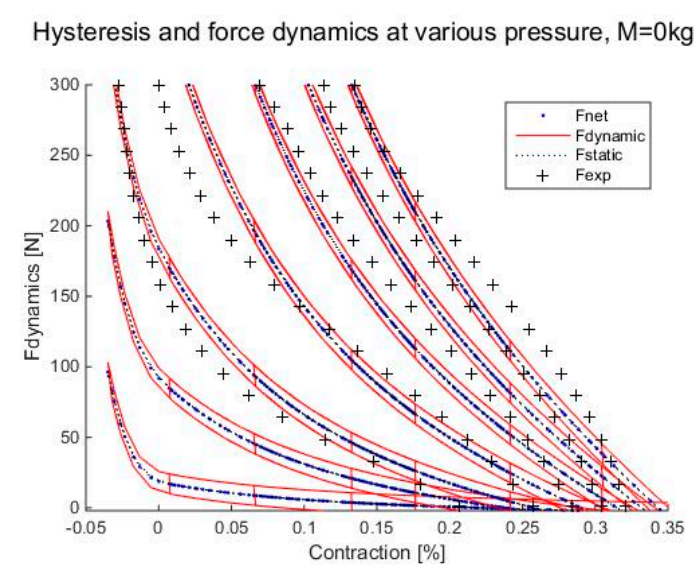

(a)

Hysteresis and force dynamics at various pressure, $M=20 \mathrm{~kg}$

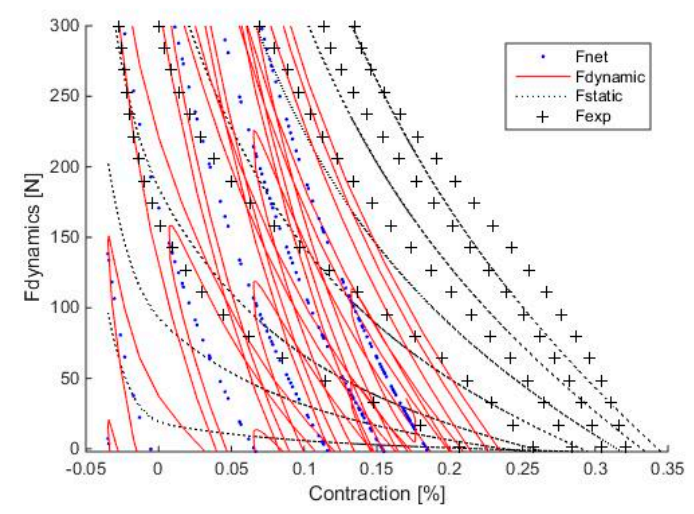

(c)

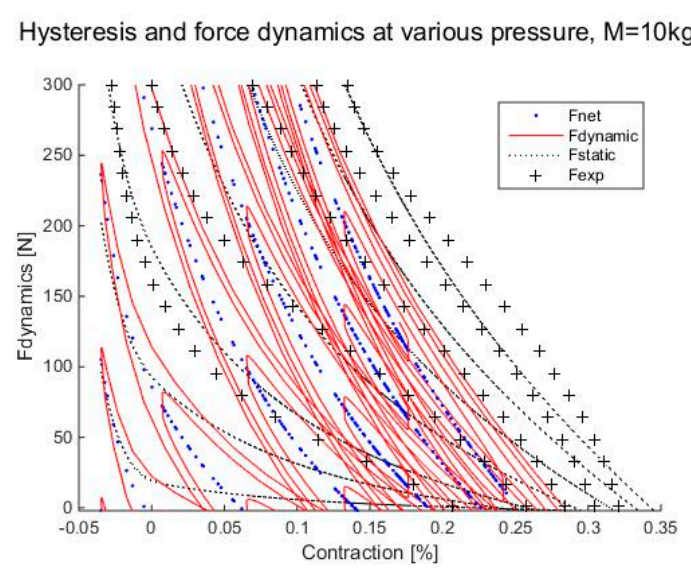

(b)

Hysteresis and force dynamics at various pressure, $M=30 \mathrm{~kg}$

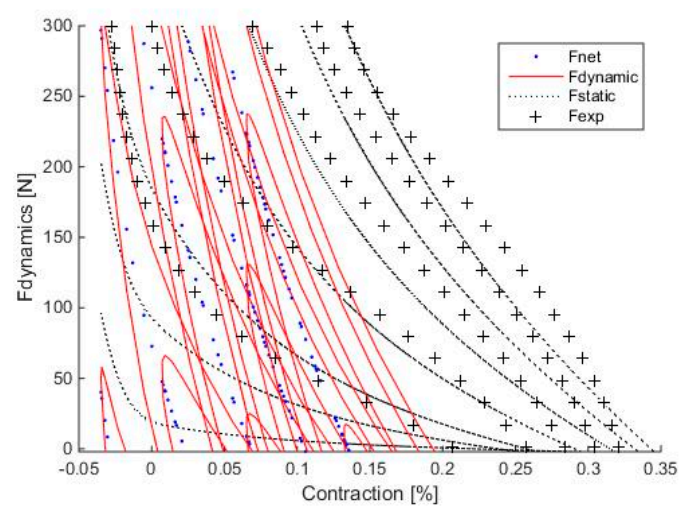

(d)

Figure 5. Force dynamics characteristics at various static load and input pressure.

\subsection{Empirical Model Simulation}

The parametric identification of the empirical model was optimized using a PSO algorithm as discussed in Section 4. The input for the model simulation was based on the actual input-output data obtained from vertical load system tests. The input data consisted of random wave of input pressure excitation for both quasi-static and quasi-rate dependency tests. All geometrical values of the McKibben muscle was carefully identified and measured. These values include initial length, diameter, braid angle, etc. The output data was the contracting length of the McKibben muscle with different dependency tests. This actual data was compared with model data obtained from the simulation. The comparison of these two data provides a fitness data (i.e., objective function or error data), which were used for parametric identification and optimization. 
Figure 6a shows existing empirical model simulation. The model simulation was carried out while considering quasi-static dependency at $0.5 \mathrm{~Hz}$ without parameter optimization. As suggested from the literature, constant coulomb and viscous friction models were implemented to compensate the hysteresis behavior of McKibben muscle. It was observed from the result that the hysteresis phenomena cannot be completely captured. This because, the friction at the contacting surface between the rubber tube and braided mesh sleeve increased with static load. Therefore, the friction model became dependent to change in static load. This behavior was similarly observed when excitation frequency of the input pressure changed (rate dependency). Figure $6 \mathrm{~b}$ shows the output of the proposed empirical model simulation. The empirical model was revised based on dynamic friction modification while considering quasi-static dependency at $0.5 \mathrm{~Hz}$. The model parameters were optimized using PSO method. It was observed from the simulation that the hysteresis phenomena behave accordingly while retaining the level of hysteresis throughout quasi-static dependency. The model verification with actual hysteresis data in Figure 2, while considering all dependencies, is shown in Figure 7.

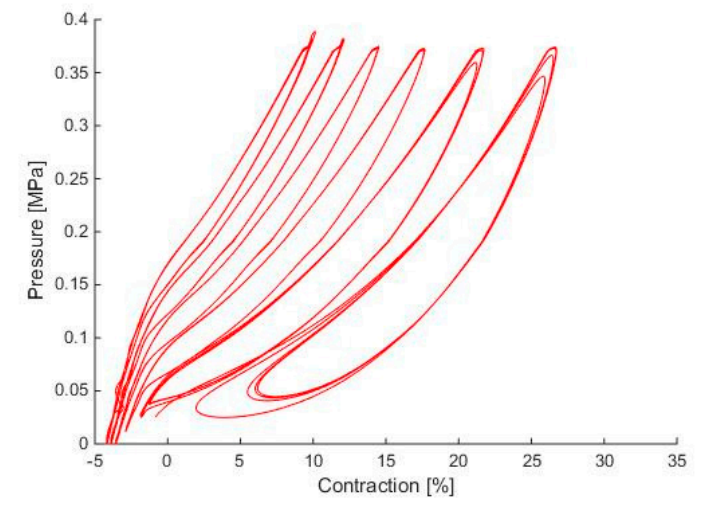

(a)

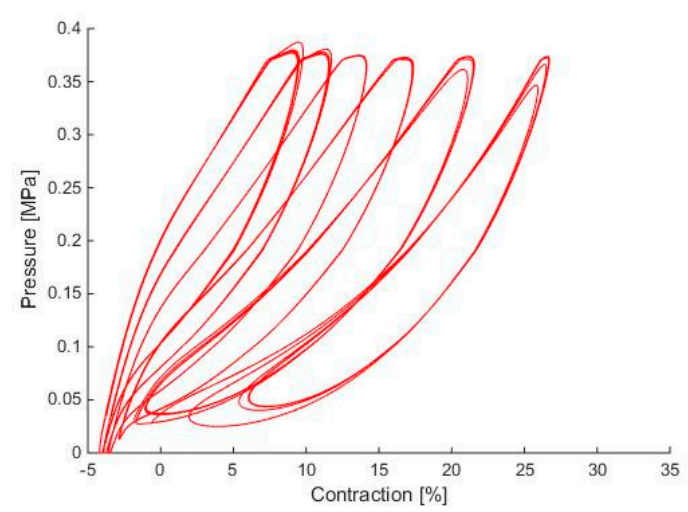

(b)

Figure 6. Model simulation: (a) existing model and (b) proposed model.

\subsection{Empirical Model Testing}

Empirical model testing was carried out to evaluate the proposed model adaptability while considering quasi-static, quasi-rate, and historic dependencies. The model performance was measured based on the correlation test evaluation. The quasi-static model testing was performed by introducing static load disturbance into the model. This generates isotonic relation of the McKibben muscle model. Contrarily, the quasi-rate model testing was performed by introducing different excitation frequency to generate the input pressure signal. This caused the contraction length, velocity, and acceleration of the McKibben muscle to change significantly. This phenomenon affects frictional behavior as described in sliding regime. Meanwhile, the historic dependence model testing was performed by generating input pressure with random wave signals. The random signals created different pattern of minor hysteresis loops with nonlocal memory behavior effect. Figure 7 shows the superimposed of proposed model with hysteresis data while considering quasi-static, quasi-rate, and historic dependencies. This result shown that the empirical modeling with modified dynamic friction model was able to perform at all dependencies. This performance also can be verified based on the model output comparison with experimental data as shown in Figure 8. It shows that the proposed model adapted with changes in quasi-static and quasi-rate dependencies; thus being able to accurately simulate the contracting length of the McKibben muscle. Furthermore, the proposed model was able to correct its path even though there were sudden changes in input pressure. The changes occurred due to the random wave signal, which produces minor hysteresis loops. Table 3 shows the correlation test of the desired trajectories and model outputs from empirical modeling. The result shown significantly high correlation values produced at lower frequencies with $(0.944 \pm 0.009)$ at $0.5 \mathrm{~Hz}$ and $(0.897 \pm 0.007)$ at $1 \mathrm{~Hz}$. Even though 
the correlation values were slightly decreased at higher frequencies with $(0.843 \pm 0.016)$ at $1.5 \mathrm{~Hz}$ and $(0.752 \pm 0.027)$ at $2 \mathrm{~Hz}$, the result was still significant. This indicated that the proposed empirical modeling was able to compensate all source of dependencies. However, there was still some error produced by the proposed model. This error cannot be avoided due to the nonlinearities inherited by the McKibben muscle were varied and complicated. These results were still not the best solution that can be produced by the proposed empirical model because the model parameters can still be improved by the PSO method.

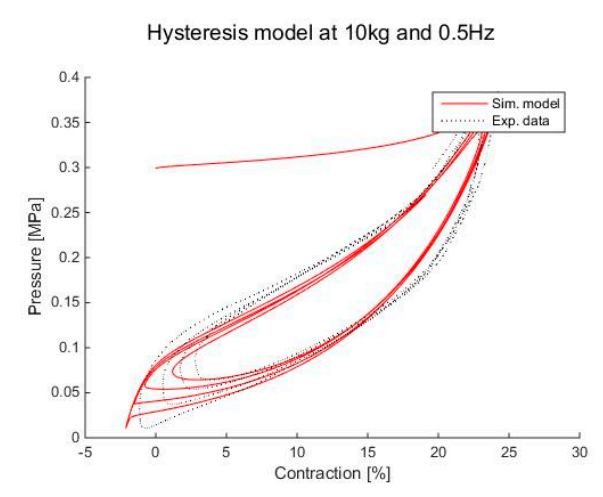

(a)

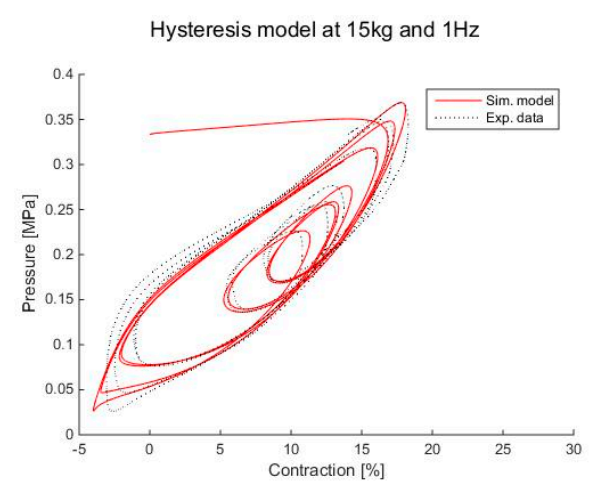

(c)

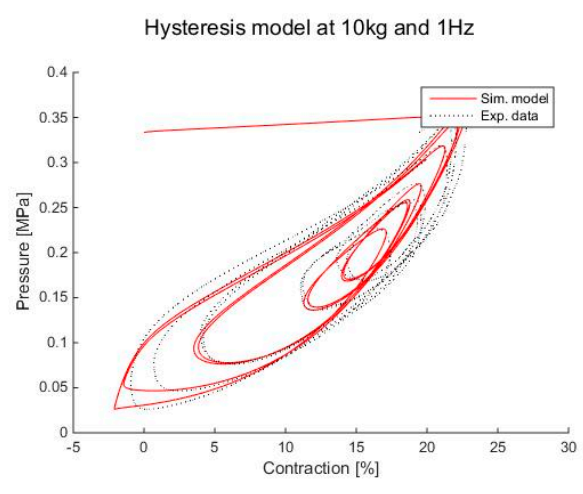

(b)

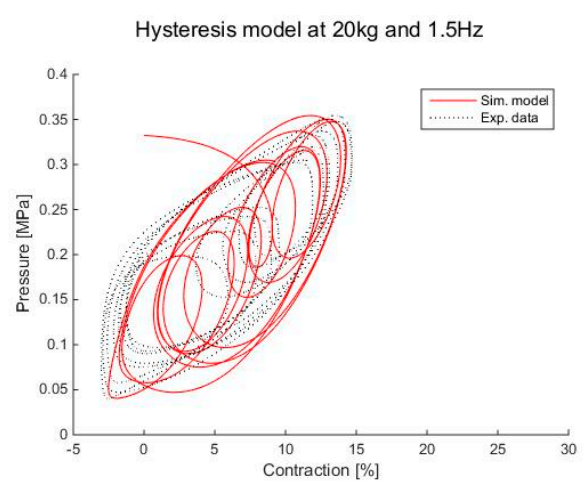

(d)

Figure 7. Hysteresis based on empirical model and experimental tests at all dependencies.

Table 3. Correlation test at all dependencies.

\begin{tabular}{lcccc}
\hline Quasi-Static $\backslash$ Rate & $\mathbf{0 . 5} \mathbf{~ H z}$ & $\mathbf{1 ~ H z}$ & $\mathbf{1 . 5} \mathbf{~ H z}$ & $\mathbf{2 ~ H z}$ \\
\hline $5 \mathrm{~kg}$ & 0.929 & 0.894 & 0.815 & 0.732 \\
$10 \mathrm{~kg}$ & 0.937 & 0.903 & 0.825 & 0.739 \\
$15 \mathrm{~kg}$ & 0.952 & 0.883 & 0.847 & 0.763 \\
$20 \mathrm{~kg}$ & 0.955 & 0.898 & 0.839 & 0.741 \\
$25 \mathrm{~kg}$ & 0.941 & 0.895 & 0.853 & 0.797 \\
$30 \mathrm{~kg}$ & 0.948 & 0.904 & 0.861 & 0.799 \\
$\bar{x} \pm \sigma$ & $0.944 \pm 0.009$ & $0.897 \pm 0.007$ & $0.843 \pm 0.016$ & $0.752 \pm 0.027$ \\
\hline
\end{tabular}


Empirical modeling at $5 \mathrm{~kg}$ and $0.5 \mathrm{~Hz}$

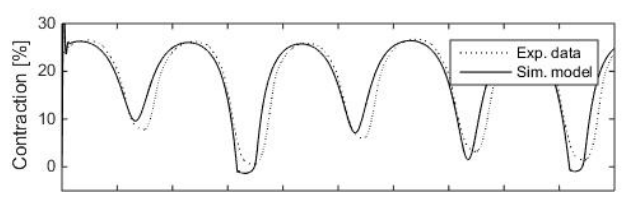

(a)

Empirical modeling at $10 \mathrm{~kg}$ and $1.5 \mathrm{~Hz}$

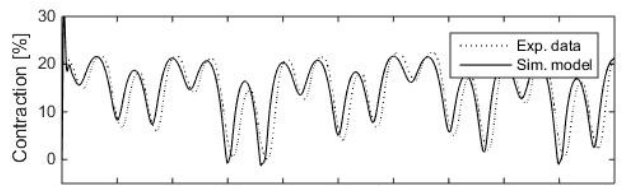

(c)

Empirical modeling at $15 \mathrm{~kg}$ and $0.5 \mathrm{~Hz}$

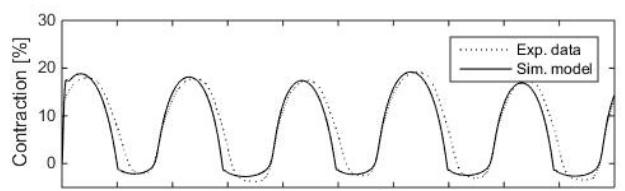

(e)

Empirical modeling at $20 \mathrm{~kg}$ and $1.5 \mathrm{~Hz}$

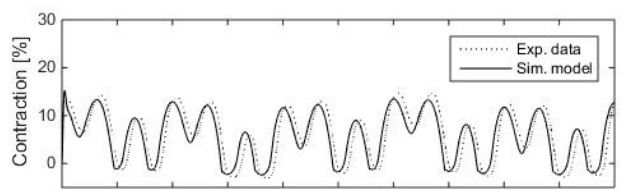

(g)

Empirical modeling at $25 \mathrm{~kg}$ and $0.5 \mathrm{~Hz}$

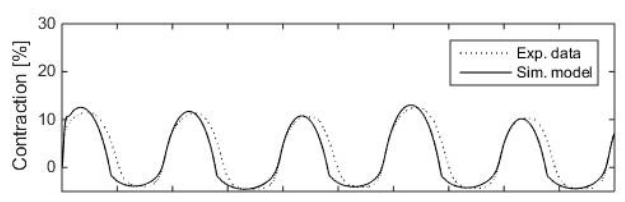

(i)

Empirical modeling at $30 \mathrm{~kg}$ and $1.5 \mathrm{~Hz}$

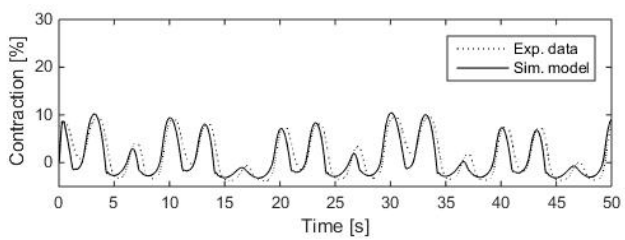

(k)
Empirical modeling at $5 \mathrm{~kg}$ and $1 \mathrm{~Hz}$

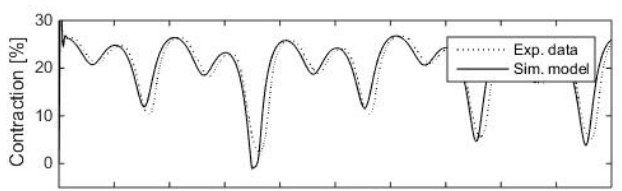

(b)

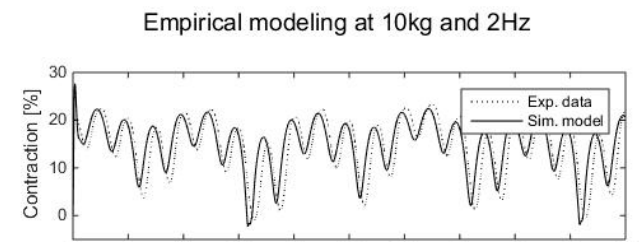

(d)

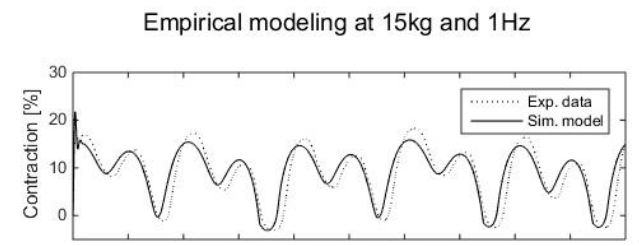

(f)

Empirical modeling at $20 \mathrm{~kg}$ and $2 \mathrm{~Hz}$

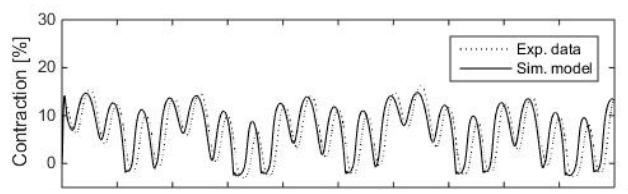

(h)

Empirical modeling at $25 \mathrm{~kg}$ and $1 \mathrm{~Hz}$

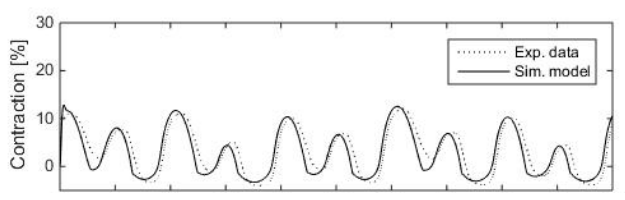

(j)

Empirical modeling at $30 \mathrm{~kg}$ and $2 \mathrm{~Hz}$

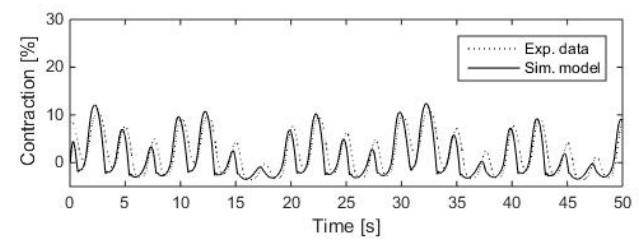

(1)

Figure 8. Empirical model testing at all dependencies $(0.5-2.0 \mathrm{~Hz})$. 


\section{Discussion}

The performance of dynamic modeling of McKibben muscle was evaluated based on comparison test of empirical modeling and hysteresis data. The hysteresis data was obtained through the experimental study, while the empirical model was modified based on the existing model presented in literature. Empirical modeling consists of two parts: the first part is the stating modeling of the contracting force model, while the second part is the dynamic modeling of the inherent hysteresis. Before performing the empirical modeling, all the initial parameters were identified and measured. Then, the simulation model was performed with parametric identification using PSO algorithm. Based on simulation analysis, the contracting force of static empirical modeling was able to characterize the quasi-static behavior within the linear region of McKibben muscle's model. The nonlinear region of the McKibben muscle which is at zero pressure condition was represented by using elasticity model. When the McKibben muscle was stretched beyond its nominal length with zero pressure intake, the McKibben consisted only of a braided mesh sleeve and the rubber tube mechanism. The force dynamic of the proposed empirical model for quasi-static dependency can be seen in Figure 5. The result shown that the proposed model was able to generate an appropriate force dynamic comparable with the one obtained through experimental study. The experimental data was obtained from the experimental test at minimum load condition. Furthermore, the model also demonstrated comparable hysteresis behavior during cyclic compression and expansion as shown in Figure 7. The model was compared with hysteresis data in Figure 2. As describe in the literature, the extracted hysteresis data was dependent on the contraction velocity of the McKibben muscle. The modified dynamic friction model was proven to be able to capture the quasi-rate behavior of McKibben muscle which follows the sliding regime.

The random wave of input pressure was used to test the effect of the nonlocal memory or historic dependency on the proposed empirical model. This random input data consisted of various contraction patterns during cyclic contraction and expansion of the McKibben muscle. By employing random input data, the ability of the model to redefine its path towards required value can be clarified. In addition, the reliability and adaptability of the dynamic model of hysteresis also can be verified. The ability to convert the present path into the desired path is what defines the history dependency. The influence of the nonlocal memory causes the dynamic modeling of McKibben muscle complicated and difficult to be achieved. The proposed model testing while considering quasi-static, quasi-rate and historic dependencies were shown in Figure 8. These tests were accomplished by generating the random wave of input pressure with different excitation frequencies $(0.5-2 \mathrm{~Hz})$, and were then performed with various static loading $(0-300 \mathrm{~N})$. Based on the result, the proposed model was able to simulate the desired contracting length precisely with all sources of dependencies. The performance of the proposed empirical model was evaluated using a correlation test. Table 3 shows the correlation test of the proposed empirical model at all dependencies. The correlation test indicated the model performance with high mean correlation value and low standard deviation. The model was performed very well at lower frequencies with correlation values of $(0.944 \pm 0.009)$ at $0.5 \mathrm{~Hz}$ and $(0.897 \pm 0.007)$ at $1 \mathrm{~Hz}$. For higher frequencies, the result was still significant with slightly decreasing correlation values of $(0.843 \pm 0.016)$ at $1.5 \mathrm{~Hz}$ and $(0.752 \pm 0.027) 2 \mathrm{~Hz}$. Although the modification model and correction function were presented, there were some errors remaining due to the high nonlinearities of McKibben muscle that cannot be captured. These remaining errors could easily be eliminated with intelligent control strategies.

\section{Conclusions}

In conclusion, empirical modeling of the McKibben with quasi-static, quasi-rate, and historic dependencies was presented. A modification was made on the existing empirical model in literature to accurately capture the inherent hysteresis of McKibben muscle. Additional correction functions were proposed to increase the adaptability of the dynamic friction model when tested with all dependencies. The initial and boundary conditions specifying nonlinearities of the McKibben muscle during the cyclic 
contraction and expansion were identified and measured. These values were essential to enhance the parametric identification using the PSO algorithm. Based on the obtained results, the implemented static modeling was able to capture the quasi-static behavior of McKibben muscle. The approximate static contracting force was comparable within the linear region; while the nonlinear region was compensated by implementation of elasticity model at zero pressure condition. The modification on dynamic friction model was able to capture the quasi-rate behavior of the McKibben muscle. When induced with different excitation frequencies, the proposed model was able to precisely follow the desired path of the contracting length similar with actual data. The final results show that model outputs were comparable to the hysteresis data. The correlation test was used to clarify the model testing at all dependencies with scientific justification. In addition, the McKibben muscle also exhibits a nonlocal memory behavior and it is historic dependence. If the contraction or expansion of the McKibben muscle failed to reach its final value its path changes depending on the past values of contracting length, force, and pressure. Therefore, in the dynamic modeling of hysteresis, the nonlocal memory plays a major role in determining the adaptability of the proposed model. There will be a sudden increase in the contracting force and pressure during cyclic contraction-expansion process without considering this dependency. This research finding will be important in improving lots of control system areas which implement McKibben muscle and equivalent actuators in the field of rehabilitation orthosis, robotics, biomedical etc.

Author Contributions: Conceptualization: M.A.M.D.; Formal analysis: M.A.M.D.; Funding acquisition: S.-i.Y.; Investigation: M.A.M.D.; Methodology: M.A.M.D.; Resources: M.A.M.D.; Software: M.A.M.D.; Supervision: S.-i.Y.; Validation: M.A.M.D.; Writing—original draft: M.A.M.D.; Writing—review \& editing: S.-i.Y..

Funding: This research was funded by the Shibaura Institute of Technology and Tier 1 Grant Q.J130000.2524.20H25, Universiti Teknologi Malaysia (UTM).

Acknowledgments: This research study was also supported by the help of Neuro-Rehabilitation laboratory members, Shibaura Institute of Technology.

Conflicts of Interest: The authors declare no conflicts of interest.

\section{References}

1. Dzahir, M.A.M.; Yamamoto, S.I. Recent trends in lower-limb robotic rehabilitation orthosis: control scheme and strategy for pneumatic muscle actuated gait trainers. Robotics 2014, 3, 120-148. [CrossRef]

2. Dzahir, M.A.M.; Yamamoto, S.I. Design and Evaluation of the AIRGAIT Exoskeleton: Leg Orthosis Control for Assistive Gait Rehabilitation. J. Robot. 2013, 2013, 535106. [CrossRef]

3. Zhang, Z.; Philen, M. Pressurized artificial muscles. J. Intell. Mater. Syst. Struct. 2011, 23, 255-268. [CrossRef]

4. Kothera, C.S.; Jangid, M.; Sirohi, J.; Wereley, N.M. Experimental characterization and static modeling of McKibben actuators. J. Mech. Des. 2009, 131, 1-10. [CrossRef]

5. Daerden, F.; Lefeber, D. The concept and design of pleated pneumatic artificial muscles. Int. J. Fluid Power 2001, 2, 41-50. [CrossRef]

6. Daerden, F.; Lefeber, D. Pneumatic artificial muscles: Actuators for robotics and automation. Eur. J. Mech. Environ. Eng. 2002, 47, 10-21.

7. Inoue, K. Rubbertuators and applications for robots. In Proceedings of the International Symposium on Robotics Research, Cambridge, MA, USA, 1988; pp. 57-63.

8. Tondu, B.; Boiter, V.; Lopez, P. Naturally compliant robot-arms actuated by McKibben artificial muscles. In Proceedings of the IEEE International Conference on Systems, Man and Cybernetics, San Antonio, TX, USA, 2-5 October 1994; pp. 2635-2640.

9. Reynolds, D.B.; Repperger, D.W.; Phillips, C.A.; Bandry, G. Modeling the dynamic characteristics of pneumatic muscle. Ann. Biomed. Eng. 2003, 31,310-317. [CrossRef]

10. Hildebrandt, A.; Sawodny, O.; Neumann, R.; Hartmann, A. Cascaded control concept of a robot with two degrees of freedom driven by four artificial pneumatic muscle actuators. In Proceedings of the American Control Conference, Portland, OR, USA, 8-10 June 2005; pp. 680-685.

11. Davis, S.; Tsagarakis, N.; Canderle, J.; Caldwin, D.G. Enhanced modelling and performance in braided pneumatic muscle actuators. Int. J. Robot. Res. 2003, 22, 213-227. [CrossRef] 
12. Davis, S.; Caldwin, D.G. Braid effects on contractile range and friction modeling in pneumatic muscle actuators. Int. J. Robot. Res. 2006, 25, 359-369. [CrossRef]

13. Kerscher, T.; Albiez, J.; Zollner, J.M.; Dillmann, R. Evaluation of the dynamic model of fluid muscles using quick-release. In Proceedings of the IEEE/RAS-EMBS International Conference on Biomedical Robotics and Biomechatronics, Pisa, Italy, 20-22 February 2006.

14. Wickramatunge, K.C.; Leephakpreeda, T. Empirical modeling of pneumatic artificial muscle. In Proceedings of the International Multi-conference of Engineers and Computer Scientists, Hong Kong, China, 18-20 March 2009; Volume II.

15. Wickramatunge, K.C.; Leephakpreeda, T. Study on mechanical behaviors of pneumatic artificial muscle. Int. J. Eng. Sci. 2010, 48, 188-198. [CrossRef]

16. Krichel, S.V.; Sawodny, O.; Hildebrandt, A. Tracking control of a pneumatic muscle actuator using one servovalve. In Proceedings of the American Control Conference, Baltimore, MD, USA, 30 June-2 July 2010; pp. 4385-4390.

17. Kang, B.S.; Kothera, C.S.; Woods, B.K.S.; Wereley, N.M. Dynamic modeling of McKibben pneumatic artificial muscles for antagonistic actuation. In Proceedings of the IEEE International Conference on Robotics and Automation, Kobe, Japan, 12-17 May 2009; pp. 182-187.

18. Itto, T.; Kogiso, K. Hybrid modeling of McKibben pneumatic artificial muscle systems. In Proceedings of the IEEE International Conference on Industrial Technology, Auburn, AL, USA, 14-16 March 2011; pp. 65-70.

19. Sarosi, J. Newest approach to modeling hysteresis in the force-contraction cycle of pneumatic artificial muscle. Acta Tech. Conviniensis Bull. Eng. 2012, 5, 63-66.

20. Sarosi, J. Elimination of the hysteresis effect of PAM actuator: Modelling and experimental studies. Tech. Gaz. 2015, 6, 1489-1494.

21. Tothova, M.; Pitel, J. Dynamic model of pneumatic actuator based on advanced geometric muscle model. In Proceedings of the IEEE International conference on Computational Cybernetics, Tihany, Hungary, 8-10 July 2013; pp. 83-87.

22. Kulesza, Z. Isotonic characteristics of a pneumatic muscle actuator. Adv. Robot. 2014, 28, 833-840. [CrossRef]

23. Nuchkrua, T.; Leephakpreeda, T. Actuation of pneumatic artificial muscle via hydrogen absorption/desorption of metal hydride. Adv. Mech. Eng. 2014, 7, 364306. [CrossRef]

24. Yao, B.; Zhuo, Z.; Liu, Q.; Ai, Q. Empirical modeling and position control of single pneumatic artificial muscle. J. Control Eng. Appl. Inform. 2016, 18, 86-94.

25. Ibadi, A.A.; Meziani, S.N.; Davis, S. Valuable experimental model of contraction pneumatic muscle actuator. In Proceedings of the IEEE International Conference on Methods and Models in Automation and Robotics, Miedzyzdroje, Poland, 29 August-1 September 2016; pp. 744-749.

26. Ibadi, A.A.; Meziani, S.N.; Davis, S. Efficient structure-based models for the McKibben contraction pneumatic muscle actuator: The full description of the behavior of the contraction PMA. Actuators 2017, 6, 32. [CrossRef]

27. Ibadi, A.A.; Meziani, S.N.; Davis, S. Design, implementation and modelling of the single and multiple extensor pneumatic muscle actuators. Syst. Sci. Control Eng. 2018, 6, 80-89. [CrossRef]

28. Chou, C.P.; Hannaford, B. Measurement and modeling of McKibben pneumatic artificial muscles. IEEE Trans. Robot. Autom. 1996, 12, 90-102. [CrossRef]

29. Tondu, B.; Lopez, P. Modeling and control of McKibben artificial muscle robot actuators. IEEE Control Syst. Mag. 2000, 20, 15-38.

30. Colbrunn, R.W.; Nelson, G.M.; Quinn, R.D. Modeling of braided pneumatic actuators for robotic control. In Proceedings of the IEEE/RSJ International Conference on Intelligent Robots and Systems, Maui, HI, USA, 29 October-3 November 2001.

31. Schroder, J.; Erol, D.; Kawamura, K.; Dillmann, R. Dynamic pneumatic actuator model for a model-based torque controller. In Proceedings of the IEEE International Symposium on Computational intelligence in Robotics and Automation, Kobe, Japan, 16-20 July 2003; pp. 342-347.

32. Balasubramanian, S.; Huang, H.; He, J. Quantification of dynamic property of pneumatic muscle actuator for design of therapeutic robot control. In Proceedings of the IEEE EMBS Annual International Conference, New York, NY, USA, 30 August-3 September 2006; pp. 2734-2737.

33. Jouppila, V.; Gadsden, S.A. Modeling and identification of a pneumatic muscle actuator system controlled by an on/off solenoid valve. In Proceedings of the International fluid Power Conference, Aachen, Germany, 22-24 March 2010; pp. 1-11. 
34. Tang, R.; Liu, D. An enhanced dynamic model for McKibben pneumatic muscle actuators. In Proceedings of the Australasian Conference on Robotics and Automation, Wellington, New Zealand, 3-5 December 2012.

35. Zhong, J.; Fan, J.; Zhu, Y.; Zhao, J.; Zhai, W. Static modeling for commercial braided pneumatic muscle actuators. Adv. Mech. Eng. 2014, 2014, 425217. [CrossRef]

36. Iyer, R.V.; Tan, X. Control of hysteretic systems through inverse compensation. IEEE Control Syst. 2009, 29, 83-99. [CrossRef]

37. Oh, J.H.; Bernstein, D.S. Semilinear Duhem model for rate-independent and rate-dependent hysteresis. IEEE Trans. Autom. Control 2005, 50, 631-645.

38. Ramli, M.H.M.; Dzahir, M.A.M. A new phenomenological based rate-dependent hysteresis operator for hysteresis characterization. J. Mech. Eng. 2018, 5, 142-153.

39. Ramli, M.H.M.; Minh, T.V.; Chen, X. Pseudoextended bouc-wen model and adaptive control design with applications to smart actuators. IEEE Trans. Control Syst. Technol. 2018, 99, 1-10. [CrossRef]

40. Yoshida, H.; Kawata, K.; Fukuyama, Y.; Takayama, S.; Nakanishi, Y. A particle swarm optimization for reactive power and voltage control considering voltage security assessment. IEEE Trans. Power Syst. 2001, 99, 1232-1239.

41. Gaing, Z.L. A particle swarm optimization approach for optimum design of PID controller in AVR system. IEEE Trans. Energy Convers. 2004, 19, 384-391. [CrossRef]

42. Parsopoulos, K.E.; Vrahatis, M.N. Recent approaches to global optimization problems through Particle Swarm Optimization. Nat. Comput. 2002, 1, 235-306. [CrossRef]

43. Zheng, Y.; Zhang, L.; Qian, J.; Ma, L. Robust PID controller design using particle swarm optimizer. In Proceedings of the IEEE International Symposium on Intelligent Control, Houston, TX, USA, 5-8 October 2003.

44. Beielstein, T.B.; Parsopoulos, K.E.; Vrahatis, M.N. Design and analysis of optimization algorithms using computational statistics. Appl. Num. Anal. Comp. Math. 2004, 1, 413-433. [CrossRef]

45. Zhao, J.; Li, T.; Qian, J. Application of particle swarm optimization algorithm on robust PID controller tuning. Proceeding of the International Conference on Natural Computation, Changsha, China, 27-29 August 2005; pp. 948-957.

46. Hu, H.; Hu, Q.; Lu, Z.; Xu, D. Optimal PID controller design in PMSM servo system via particle swarm optimization. In Proceedings of the Annual Conference of IEEE Industrial Electronics Society, Raleigh, NC, USA, 6-10 November 2005.

47. Markos, S.; Kamal, H. Optimal tuning of PID controller using adaptive hybrid particle swarm optimization algorithm. Int. J. Comput. Commun. Control 2012, 7, 101-114.

48. Ikhouane, F.; Rodellar, J. A linear controller for hysteretic systems. IEEE Trans. Autom. Control 2006, 51, 340-344. [CrossRef]

49. Mayergoyz, I. Mathematical models of hysteresis. IEEE Trans. Magn. 1986, 22, 603-608. [CrossRef]

50. Xu, Q.; Kiong, K. Advanced Control of Piezoelectric Micro-/Nanopositioning Systems; Springer: New York, NY, USA, 2016.

51. Minh, T.V.; Tjahjowidodo, T.; Ramon, H.; Brussel, H.V. A new approach to modeling hysteresis in a pneumatic artificial muscle using the Maxwell-slip model. IEEE/ASME Trans. Mechatron. 2011, 16, 177-186. [CrossRef] 